\title{
Improving Clinician-Patient Communication Alleviates Stigma in Patients With Functional Dyspepsia Receiving Antidepressant Treatment
}

\author{
Xiu-Juan Yan, ${ }^{1}$ Hong-Yi Qiu, ${ }^{1}$ Qing-Qing Luo, ${ }^{1}$ Bo Wang, ${ }^{1}$ Ping Xu, ${ }^{1}$ Chen-Feng Ji, ${ }^{2}$ and Sheng-Liang Chen ${ }^{1 *}$ \\ ${ }^{I}$ Division of Gastroenterology and Hepatology, Key Laboratory of Gastroenterology and Hepatology, Ministry of Health, Renji Hospital, School \\ of Medicine, Shanghai Jiao Tong University, Shanghai Institute of Digestive Disease, Shanghai, China; and ${ }^{2}$ Department of Psychological \\ Medicine, Renji Hospital, School of Medicine, Shanghai Jiao Tong University, Shanghai, China
}

\section{Background/Aims}

Antidepressants are effective in patients with functional dyspepsia (FD). However, stigma associated with FD and antidepressants may affect treatment adherence. This study aims to explore possible communication strategies to alleviate stigma and improve adherence in patients with FD.

\section{Methods}

In this randomized, single-center, and single-blind trial, 160 patients with FD initiating antidepressant treatment were recruited. Different communication strategies were performed when prescribing antidepressants. Participants in Group 1 were told that brain is the "headquarters" of gut, and that antidepressants could act as neuromodulators to relieve symptoms of FD through regulating the functions of gut and brain. Participants in Group 2 were told that antidepressants were empirically effective for FD. Stigma scores, medication-related stigma, treatment compliance, and efficacy were analyzed.

\section{Results}

After 8-week antidepressant treatment, the proportion of patients with FD with decreased stigma scores in Group 1 was significantly higher than in Group 2 (internalized stigma: $64.10 \%$ vs $12.00 \%$; perceived stigma: $55.13 \%$ vs $13.33 \% ; P<0.01$ ). Medication-related stigma was lower in Group 1 than in Group 2 ( $P<0.05$ for 3 of 4 questions). Concurrently, patients in Group 1 had better treatment compliance $(0.71 \pm 0.25$ vs $0.60 \pm 0.25, P<0.01)$ and efficacy. In Group 1, participants with decreased post-treatment stigma scores showed better treatment compliance and efficacy than those with non-decreased scores. Decrease in stigma scores positively correlated with treatment compliance.

\section{Conclusion}

Improving knowledge of patients with FD of the disease and antidepressants via proper communication may be an effective way to alleviate stigma and promote adherence.

(J Neurogastroenterol Motil 2022;28:95-103)

Key Words

Antidepressive agents; Clinician-patient communication; Dyspepsia; Patient compliance; Stigma

Received: October 29, 2020 Revised: January 25, 2021 Accepted: March 21, 2021

(.) This is an Open Access article distributed under the terms of the Creative Commons Attribution Non-Commercial License (http://creativecommons. org/licenses/by-nc/4.0) which permits unrestricted non-commercial use, distribution, and reproduction in any medium, provided the original work is properly cited.

${ }^{*}$ Correspondence: Sheng-Liang Chen, MD, PhD

Division of Gastroenterology and Hepatology, Key Laboratory of Gastroenterology and Hepatology, Ministry of Health, Renji Hospital, School of Medicine, Shanghai Jiao Tong University, Shanghai Institute of Digestive Disease, 145 Middle Shandong Road, Shanghai 200001, China

Tel: +86-21-63200784, Fax: +86-21-63266027, E-mail: chensImd@163.com

Xiu-Juan Yan and Hong-Yi Qiu contributed equally to this work. 


\section{Introduction}

Functional dyspepsia (FD) is one of the most prevalent functional gastrointestinal disorders (FGIDs). It is characterized by symptoms including early satiation, postprandial fullness, epigastric pain and burning, which are unexplained after routine clinical examinations. ${ }^{1}$ The efficacy of regular first line treatment, such as antiacid and prokinetic agents, are currently unsatisfactory for refractory $\mathrm{FD}^{2}$

In the Rome IV criteria, FGIDs have been redefined as disorders of the gut-brain axis. ${ }^{3}$ Psychological factors (such as depression and anxiety) have been demonstrated to be associated with FD. ${ }^{1}$ The American College of Gastroenterology and the Canadian Association of Gastroenterology guidelines on dyspepsia recommend that patients with FD who are not responding to proton pump inhibitors (PPIs) or Helicobacter pylori eradication therapy should be offered tricyclic antidepressants or psychological therapies. ${ }^{4}$ Psychological medications, such as tricyclic antidepressants, and noradrenergic and specific serotonin antidepressants, have been shown to be effective in the treatment of FD. ${ }^{3,5-8}$ Recent studies reported that selective serotonin reuptake inhibitors, serotonin and noradrenaline reuptake inhibitors are also beneficial to patients with $\mathrm{FD}^{3,8,9}$

However, compliance with antidepressant treatment is handicapped due to stigma. ${ }^{10-13}$ Our recent research found that stigma associated with FD and antidepressant medications could interfere with treatment adherence. ${ }^{10} \mathrm{~A}$ large proportion of FD patients refuse to take antidepressants and report concerns such as "my family says these drugs are for insane people" or "these drugs will make me lose my mind." These are typical expressions of stigma. Stigma is the negative stereotypes or discrimination toward a particular population. ${ }^{11}$ There are 2 types of stigma that may be an obstacle to adherence. Individuals' endorsement of various stereotypes toward their condition is classified as internalized stigma. The negative stereotypes displayed by others is classified as perceived stigma. ${ }^{11}$ It is essential to explore possible strategies to alleviate stigma in patients with FD.

It has been reported that there is a "communication gap" between patients with FGIDs and doctors. ${ }^{14}$ Many patients with FD hold the belief that their dyspepsia symptoms are caused by "something wrong in the stomach" rather than a "functional" property. Collins et al found that most patients with FGIDs even do not acknowledge their diagnosis. ${ }^{14}$ The antidepressant medications carry more misunderstanding. It has been reported that patients with FGIDs hold negative attitudes toward psychological medications. ${ }^{15}$
These phenomena are worse in the Chinese population because many Chinese patients do not accept psychological diagnoses and treatment. ${ }^{16,17}$ The existence of communication gap could influence treatment adherence. ${ }^{14}$ Furthermore, insufficient communication or the callous attitude of doctors could also result in complaints of dissatisfaction. ${ }^{18}$ Based on the above reasons, clinician-patient communication may be an important interventional target to alleviate stigma and improve treatment adherence.

It has been reported that physicians' attention to psychological barriers in antidepressant treatment, such as stigma, may improve medication compliance and affect the course of illness. ${ }^{12,19}$ Therefore, it is critical to find a way to fill the doctor-patient communication gap and reduce stigma in patients with refractory FD. A previous systematic review suggested that clinician-patient communication may be important in increasing cooperation in mental health care. ${ }^{20}$ However, it is unclear whether clinician-patient communication affects stigma in patients with FD. Therefore, this study is designed to explore clinician-patient communication strategies that could alleviate stigma and improve compliance with antidepressant therapy in patients with refractory FD.

\section{Materials and Methods}

\section{Participants}

This study was conducted in accordance with the ethical principles of the Declaration of Helsinki and local regulations (Trial registration No. NCT03625674, ClinicalTrials.gov). Ethical approval was obtained from the Ethics Committee of Renji Hospital (Approval No. 2018-082). Every enrolled participant signed the informed consent prior to the study.

Refractory FD patients who did not respond to 4-week regular first line treatment (such as antacids and prokinetics) and needed to initiate psychoactive medication treatment for the first time were enrolled from the outpatient department from January 2018 to February 2020. Symptom nonresponse was defined as less than half of symptom improvement after first line treatment. The inclusion criteria were as follows: age of 18-70 years; met the Rome IV FD criteria; Generalized Anxiety Disorder-7 (GAD-7) scale $\geq 5$ or Patient Health Questionnaire-9 (PHQ-9) depression scale $\geq 5$. The exclusion criteria included organic digestive diseases; presence of Helicobacter pylori infection; severe psychological symptoms or cognitive disorders; GAD-7 $\geq 15$ or PHQ-9 $\geq 15$; breastfeeding or pregnancy; history of gastric surgery; use of PPIs, prokinetic agents or other medications that might affect gastric function within 
1 month; and previous use of psychoactive agents.

\section{Study Design}

We performed a randomized, single-center, single-blind, controlled study to explore possible ways to alleviate stigma and improve medication adherence through appropriate clinician-patient communication. Physicians from the gastrointestinal (GI) department and psychological department met the participants together and chose appropriate antidepressant agents for each patient. Stratified randomization was conducted according to the antidepressants they took. Thus, the enrolled patients who were prescribed with the same antidepressant were randomized into 2 groups based on random number lists generated from a computer. Two doctors were trained to communicate with the enrolled patients according to the following strategies every 2 weeks when the patient received their antidepressant agents. The patients in Group 1 were told: (1) Brain is the "headquarters" of GI tract; their FD symptoms are caused by dysfunction of brain and GI tract, rather than a sole GI or psychological problem. (2) Psychoactive drugs act as neuromodulators to relieve FD symptoms through regulating functions of GI tract and brain. (3) The dose of antidepressants used in FD patients is lower than those used in patients with anxiety or depression. The patients in Group 2 were told that clinical evidence has shown antidepressants are useful in alleviating their symptoms. The information of dosage and possible adverse effects were provided to the participants in both groups. The duration of doctor-patient communication (about 20 minutes) were similar in the 2 groups. The allocation information was blind to data collectors.

The treatment period for the participants was 8 weeks. Demographic data, dyspeptic and psychological symptom scales, and stigma scales were assessed at enrollment. Medications were prescribed every 2 weeks, with medication adherence, stigma scales, a 4-question survey, ${ }^{21}$ and symptom scales being assessed. The primary endpoint for this study was medication adherence evaluated by medication possession ratio (MPR). The secondary endpoints were stigma assessed by internalized stigma scale (IS) and perceived stigma scale (PSS) scores, and symptom relief assessed by the Leeds Dyspepsia Questionnaire (LDQ), GAD-7, and PHQ-9 scales.

\section{Outcome Measures}

IS and PSS are widely used in assessing the degree of internalized or perceived stigma. ${ }^{11}$ The term "mental illness" was replaced with "FD" in the scales. Each item in IS scale was ranked on a 4-point scale (1-4). The final IS score was calculated as the mean score of all the 24 items. Higher scores indicate worse internalized stigma: scores $\leq 2$ were labeled as "minimal stigma," scores $>2$ and $\leq 2.5$ were "mild stigma," scores $>2.5$ and $\leq 3$ were "moderate stigma," and scores $>3$ were "severe stigma." ${ }^{22}$ The PSS had 10 items ranked on a 5-point scale (1-5). The final PSS score was calculated as the mean score of the 10 items. Higher scores indicated worse perceived stigma.

Because IS and PSS scores cannot distinguish disease-related stigma and medication-related stigma, antidepressant-related stigma was evaluated every 2 weeks using a 4-question survey adapted from a previous study. ${ }^{21}$ The following questions were answered with "yes" or "no" by the participants: when taking the antidepressant agents, (1) I feel ashamed; (2) I do not feel comfortable to tell friends or family; (3) I do not feel okay if people in community know; and (4) I do not want to tell people at job. ${ }^{21}$ The percentage of the answer "yes" for each question was calculated in each group.

The LDQ scale was used to assess dyspeptic symptoms. ${ }^{23}$ The scoring criteria were as follows: $0-4$, very mild dyspepsia; $5-8$, mild dyspepsia; 9-15, moderate dyspepsia; and $>15$, severe or very severe dyspepsia.

The GAD-7 and PHQ-9 questionnaires were used to evaluate the psychological status of enrolled patients. The GAD-7 scale, which is used to screen generalized anxiety disorder ${ }^{24}$ consists of 7 items on a 4-point questionnaire (0-3). The final GAD-7 scores were calculated as the sum of the 7 items. The scoring criteria were as follows: 0-4, no anxiety; 5-9, mild anxiety; 10-14, moderate anxiety; and $\geq 15$, severe anxiety. The scores of PHQ-9, a 10-item scale used to screen for depression, ${ }^{25}$ was calculated as the sum of the 10 items. The scoring criteria were as follows: 0-4, none or minimal depression; 5-9, mild depression; 10-14, moderate depression; 15-19, moderately severe depression; and $\geq 20$, severe depression.

Medication adherence was evaluated with MPR. MPR is defined as the sum of the supplied medication divided by the number of days between the first and the last refill. ${ }^{26}$ Higher MPR scores indicate better compliance. The patients were asked to keep a medication diary for calculating the MPR.

\section{Statistical Methods}

All enrolled participants were included in the adherence analyses. The MPR values of patients who were lost in the first followup were assigned as zero. Calculation of symptom improvement was based on the subjects who had at least 1 follow-up. The last observation carried forward method was used in cases of pre-mature study termination.

Sample size calculation was based on the assumption that the MPR values after 8 -week treatment would be 0.71 in Group 1 
and 0.60 in Group 2 with $\alpha=0.05$ (2-tailed) and $\beta=0.20$. The estimated sample size was 79 patients per group. Therefore, a minimal of 158 participants were needed in order to prove that MPR in Group 1 was significantly different from that in Group 2.

SPSS version 22 software (IBM, Armonk, NY, USA) was

Table 1. Baseline Characteristics of the Enrolled Participants $(\mathrm{n}=80)$

\begin{tabular}{lcc}
\hline \multicolumn{1}{c}{ Characteristics } & Group 1 & Group 2 \\
\hline Age (yr) & $45.64 \pm 12.64$ & $46.25 \pm 11.04$ \\
Male:female ratio & $26: 54$ & $25: 55$ \\
LDQ & $13.34 \pm 3.14$ & $13.68 \pm 2.82$ \\
GAD-7 & $6.62 \pm 2.75$ & $6.56 \pm 2.01$ \\
PHQ-9 & $5.80 \pm 2.92$ & $5.91 \pm 2.70$ \\
IS & $1.60 \pm 0.39$ & $1.64 \pm 0.40$ \\
PSS & $1.61 \pm 0.46$ & $1.61 \pm 0.38$ \\
\hline
\end{tabular}

LDQ, leeds dyspepsia questionnaire; GAD-7, generalized anxiety disorder-7 scale; PHQ-9, patient health questionnaire-9 depression scale; IS, internalized stigma scale; PSS, perceived stigma scale.

Data are presented as mean $\pm \mathrm{SD}$.

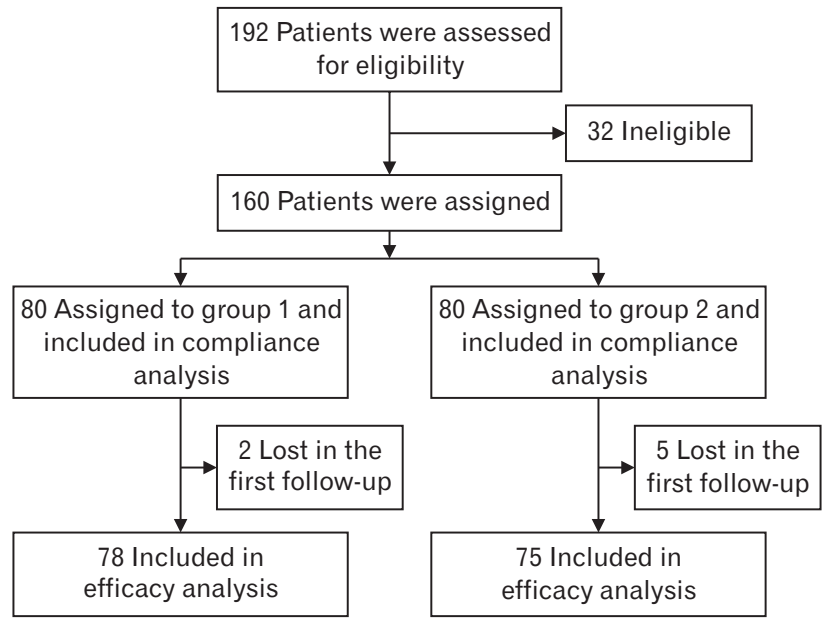

Figure 1. Flowchart of screening and recruitment of study subjects. used to perform statistical analyses. Data from normally distributions were presented as mean $\pm \mathrm{SD}$. Skewed distributed data were presented as medians and analyzed with nonparametric statistics. Differences between means before and after treatment were analyzed using paired $t$ test. Mann-Whitney test and $t$ test were used to compare the differences between groups. Correlations were assessed with Spearman correlation analyses. $P<0.05$ was considered statistically significant.

\section{Results}

\section{Participant Characteristics}

A total of 160 patients were enrolled from the GI outpatient department. Demographic and baseline clinical characteristics were well balanced between the 2 groups (Table 1). Seven patients who were lost in the first follow-up (thus had no post-treatment data) were excluded from efficacy analyses (Fig. 1). Antidepressants taken in each group were listed in Table 2.

\section{Stigma Scores Before and After Treatment}

Stigma scores before treatment were well balanced between the 2 groups. After 8-week treatment, patients with FD in Group 1 showed lower stigma scores compared with those in Group 2

Table 2. Antidepressants Taken in Each Group

\begin{tabular}{lrc}
\hline \multicolumn{1}{c}{ Drugs } & Group 1 & Group 2 \\
\hline Mirtazapine & 21 & 21 \\
Fluoxetine & 16 & 15 \\
Sertraline & 15 & 16 \\
Paroxetine & 13 & 13 \\
Citalopram & 9 & 10 \\
Venlafaxine & 6 & 5 \\
Total & 80 & 80 \\
\hline
\end{tabular}

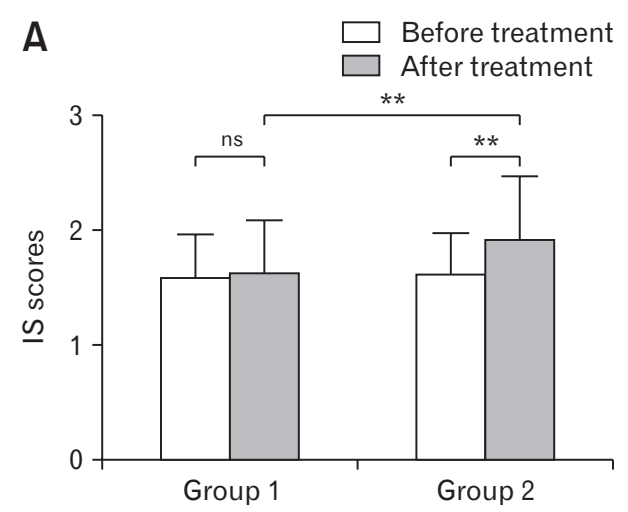

Figure 2. Effect of different communication strategies on internalized stigma scale (IS) and perceived stigma scale (PSS) scores in patients with functional dyspepsia (FD). (A) IS and (B) PSS scores before and after treatment in Group $1(\mathrm{n}=78)$ and Group $2(\mathrm{n}=75) .{ }^{* *} \mathrm{P}<$ 0.01 . 
Table 3. Number of Patients With Functional Dyspepsia With Altered Stigma Scores After Treatment

\begin{tabular}{clcc}
\hline $\begin{array}{c}\text { Stigma } \\
\text { scores }\end{array}$ & Change of scores & $\begin{array}{c}\text { Group 1 } \\
(\mathrm{n}=78)\end{array}$ & $\begin{array}{c}\text { Group 2 } \\
(\mathrm{n}=75)\end{array}$ \\
\hline \multirow{2}{*}{ IS } & Decreased & $50(64.10)^{\mathrm{a}}$ & $9(12.00)$ \\
& Unaltered & $7(8.97)$ & $5(6.67)$ \\
& Elevated & $21(26.92)^{\mathrm{a}}$ & $61(81.33)$ \\
PSS & Decreased & $43(55.13)^{\mathrm{a}}$ & $10(13.33)$ \\
& Unaltered & $5(6.41)$ & $4(5.33)$ \\
& Elevated & $30(38.46)^{\mathrm{a}}$ & $61(81.33)$ \\
\hline
\end{tabular}

IS, internalized stigma scale; PSS, perceived stigma scale.

${ }^{a} P<0.01$ ( $\chi^{2}$ test).

Data are presented as $n(\%)$.

Table 4. Medicine-associated Stigma After Treatment

\begin{tabular}{lccc}
\hline \multicolumn{1}{c}{ Questions } & $\begin{array}{c}\text { Group 1 } \\
(\mathrm{n}=78)\end{array}$ & $\begin{array}{l}\text { Group 2 } \\
(\mathrm{n}=75)\end{array}$ & $\begin{array}{r}\text { P-value } \\
\left(\chi^{2} \text { test }\right)\end{array}$ \\
\hline $\begin{array}{l}\text { Feel ashamed } \\
\text { Do not feel comfortable to tell }\end{array}$ & $\begin{array}{c}17(21.79) \\
\quad \text { friends or family }\end{array}$ & $32(42.67)$ & 0.010 \\
$\begin{array}{l}\text { Do not feel okay if people in } \\
\text { community know }\end{array}$ & $52(66.67)$ & $69(46.67)$ & 0.064 \\
$\begin{array}{l}\text { Do not want to tell people at job } \\
\text { Do }\end{array}$ & $46(58.97)$ & $60(80.00)$ & 0.008 \\
\hline
\end{tabular}

Data are presented as $\mathrm{n}(\%)$.

( $P<0.01$; Fig. 2). In Group 1, 50 participants (64.10\%) showed decreased post-treatment IS scores, 7 participants $(8.97 \%)$ showed unaltered scores and 21 participants $(26.92 \%)$ showed elevated IS scores after treatment. As for PSS scores, 43 participants $(55.13 \%)$ showed decreased, 5 participants $(6.41 \%)$ showed unaltered, and 30 participants $(38.46 \%)$ showed elevated scores after treatment. However, in Group 2, 9 participants $(12.00 \%, P<0.01$ vs Group 1) showed decreased post-treatment IS scores, 5 participants (6.67\%) showed unaltered, and 61 participants $(81.33 \%, P<0.01$ vs Group 1) showed elevated IS scores after treatment. Ten participants $(13.33 \%, P<0.01$ vs Group 1$)$ showed decreased posttreatment PSS scores, 4 participants $(5.33 \%)$ showed unaltered scores, and 61 participants $(81.33 \%, P<0.01$ vs Group 1) showed elevated PSS scores after treatment (Table 3). IS and PSS scores were elevated in Group 2 (increased by $18.38 \%$ and $23.57 \%$, respectively; $P<0.01$ vs baseline), while the mean scores in Group 1 showed no obvious change after treatment (Fig. 2).

Assessment of medication-associated stigma with the 4-question survey revealed that patients in the 2 groups had different levels of antidepressant medication-related stigma after treatment. The percentage of patients answering “yes" for question 1 (feel ashamed:

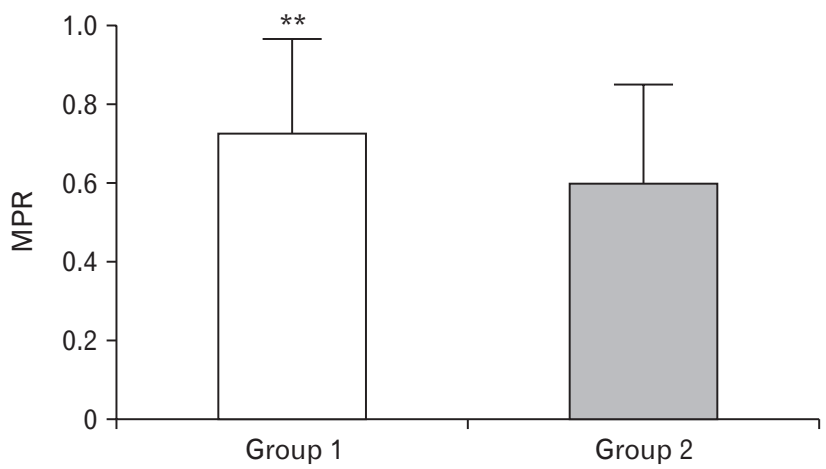

Figure 3. Medication possession ratio (MPR) values of all enrolled patients after 8 -week antidepressant treatment $(\mathrm{n}=80)$. ${ }^{* *} P<0.01$.

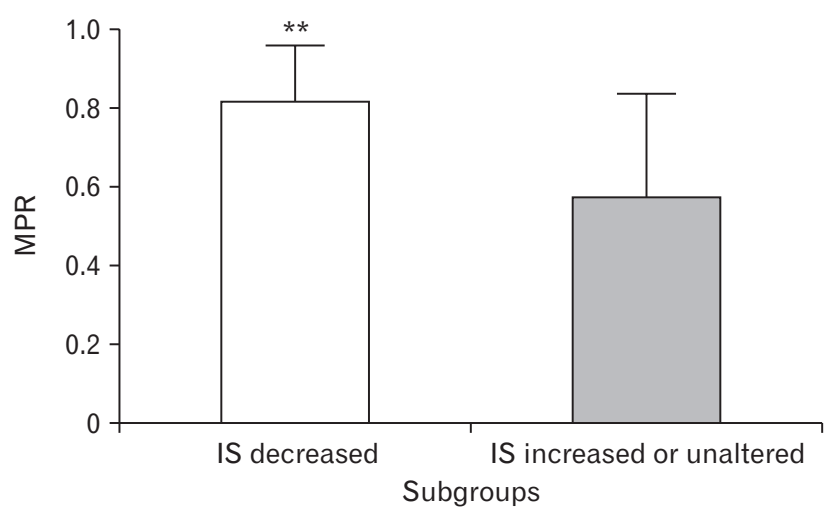

Figure 4. Subgroup analysis of medication possession ratio (MPR) in Group 1 according to changes in internalized stigma scale (IS) scores. $* * P<0.01$ (Mann-Whitney test).

$21.79 \%$ vs $42.67 \%$ ), question 3 (do not feel okay if people in community know: $66.67 \%$ vs $92.00 \%$ ), and question 4 (do not want to tell people at job: $58.97 \%$ vs $80.00 \%$ ) in Group 1 were significantly lower than in Group $2(P<0.05$; Table 4$)$. The percentage of patients answering "yes" for question 2 (do not feel comfortable to tell friends or family) showed no significant difference between the 2 groups (Table 4).

\section{Medication Adherence}

After 8-week treatment, MPR values of the participants in Group 1 were significantly higher than in Group $2(0.71 \pm 0.25$ vs $0.60 \pm 0.25, P<0.01$; Fig. 3$)$. These results suggested that patients' adherence to antidepressants in Group 1 was better than in Group 2. We then analyzed the participants in Group 1 according to the changes in IS scores after treatment. We found that MPR values of participants with decreased IS scores after treatment was significantly higher than those with unaltered or elevated IS scores 
after treatment $(P<0.01$; Fig. 4$)$.

In the correlation analyses, we found that the decrease of IS and PSS scores after treatment were positively associated with MPR values $(P<0.001$; Table 5$)$.

\section{Treatment Efficacy}

The mean LDQ, GAD-7, and PHQ-9 scores of both groups were reduced compared with baseline after the 8-week treatment $(P<0.05$; paired $t$ test). Moreover, patients in Group 1 showed a greater decrease in LDQ, GAD-7, and PHQ-9 scores than in Group 2 (LDQ: $P<0.01$, GAD-7 and PHQ-9: $P<0.05$; Fig. 5). Sixty-nine patients (88.46\%) in Group 1 and 45 patients $(60.00 \%)$ in Group 2 showed improvement in LDQ scores.

Subgroup analyses of symptom scores in Group 1 showed that the scores of dyspeptic, anxiety, and depressive symptoms in participants with decreased post-treatment IS scores displayed a greater decrease than those of participants with unaltered or elevated posttreatment IS scores $(P<0.01$ for LDQ and PHQ-9, $P<0.05$ for GAD-7; Fig. 6).

\section{Safety Analyses}

Seven patients in Group $1(7 / 78,8.97 \%)$ and 9 patients in Group $2(9 / 75,12.00 \%)$ complained of discomforts including insomnia, sleepiness, dizziness, and skin rash during treatment $(P>$

Table 5. Correlation Between the Decrease of Stigma Scores and Medication Possession Ratio Values

\begin{tabular}{ccccccc}
\hline \multirow{2}{*}{$\begin{array}{c}\text { Decrease of } \\
\text { stigma scores }\end{array}$} & \multicolumn{2}{c}{ Group 1 $(\mathrm{n}=78)$} & & \multicolumn{2}{c}{ Group 2 $(\mathrm{n}=75)$} \\
\cline { 2 - 3 } \cline { 5 - 6 } & \multicolumn{2}{c}{$P$} & & & $r$ & $P$ \\
\hline Decrease of IS & 0.601 & $<0.001$ & & 0.659 & $<0.001$ \\
Decrease of PSS & 0.503 & $<0.001$ & & 0.575 & $<0.001$ \\
\hline
\end{tabular}

Spearman correlation analysis.

IS, internalized stigma scale; PSS, perceived stigma scale.
0.05; Supplementary Table). All these adverse effects were mild. No severe adverse events occurred during the treatment.

\section{Discussion}

Stigma is present in many GI disorders such as FD, irritable bowel syndrome, and inflammatory bowel disease. ${ }^{10,27,28}$ Treatment adherence and efficacy of antidepressants are greatly hindered by stigma associated with diseases and antidepressants. ${ }^{10,12}$ In this study, we demonstrated that proper physician-patient communication strategies may alleviate stigma and improve adherence to antidepressant treatment in patients with refractory FD.

We first explored whether different physician-patient communication strategies could affect stigma in refractory FD patients receiving antidepressant treatment. Our previous observational study showed that stigma scores increased significantly after initiating antidepressants in FD patients from our outpatient department. ${ }^{10}$ Besides, we found that the types of antidepressants showed no sig-

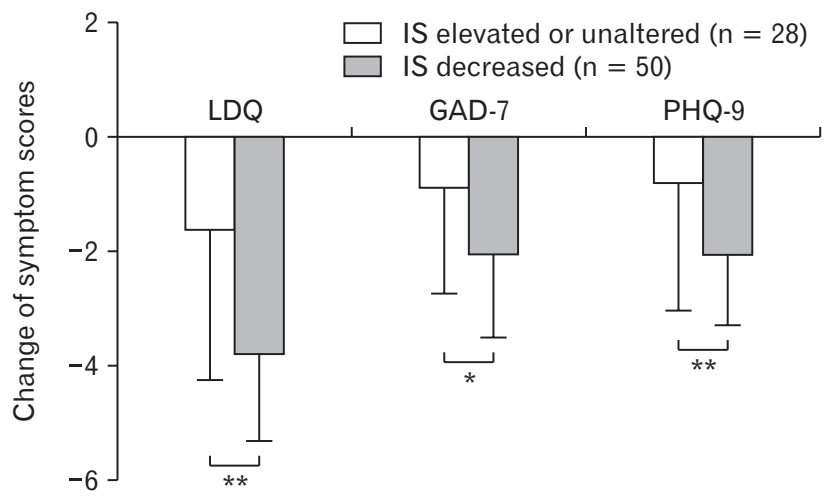

Figure 6. Subgroup analysis of symptom scores in Group 1 based on change of internalized stigma scale (IS) scores. LDQ, leeds dyspepsia questionnaire; GAD-7, generalized anxiety disorder scale; PHQ-9, patient health questionnaire depression scale. ${ }^{*} P<0.05,{ }^{*} P<0.01$.
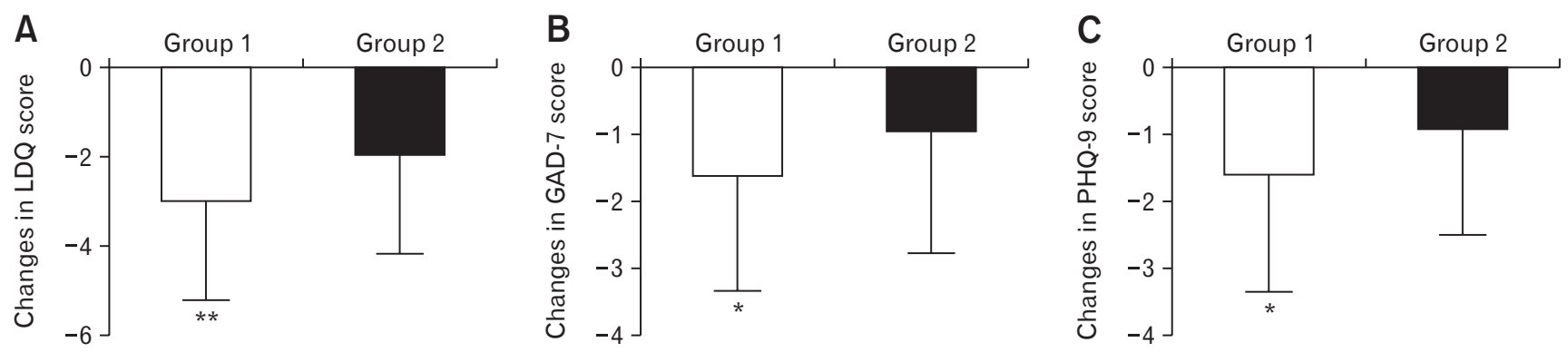

Figure 5. Mean changes from baseline symptom scores of patients with functional dyspepsia (FD) in Group 1 ( $\mathrm{n}=78)$ and Group 2 ( $\mathrm{n}=75$ ). Changes in the (A) Leeds Dyspepsia Questionnaire (LDQ), (B) Generalized Anxiety Disorder-7 (GAD-7) scale, and (C) Patient Health Questionnaire-9 (PHQ-9) depression scale scores. ${ }^{*} P<0.05,{ }^{* *} P<0.01$. 
nificant correlation with stigma, perhaps because patients with FD classify them all as psychological drugs. ${ }^{10}$ Thus in this study we performed different communication strategies with patients with FD who needed to initiate antidepressants. We found that $64.10 \%$ of patients in Group 1 had decreased post-treatment IS scores, while this proportion was only $12.00 \%$ in Group 2 . As it is hard to distinguish the disease-related stigma and antidepressant-related stigma in IS and PSS scores, we used the 4-question survey and found that the percentage of patients with antidepressant-related stigma (except for question 2) in Group 1 was significantly lower than in Group 2. These results suggest that proper communication could relieve stigma attached to FD and antidepressant.

Because many patients may be unfamiliar with FGIDs and psychological diagnosis, a previous study on depression suggested that physicians should understand the meanings of illness from the patients' perspective and communicate with them using a language that the patients can understand. ${ }^{16}$ In this study, the mechanisms of antidepressants were interpreted to patients as relieving symptoms by "restoring balance to the nervous system," or by "tonifying or strengthening the energy of the brain." ${ }^{\prime 16}$ Thus, in our study we tried to explain "gut-brain axis" in plain language to patients with $\mathrm{FD}$, and the results showed a promising effect in reducing stigma. Our communication strategy included 3 parts. The first and second parts were focused on explanation of the symptoms and antidepressant agents, which helped patients with FD to understand their diagnosis and treatment. The last part was explanation of the different doses adopted in GI and in the psychological department, which is also important because it could make the patients with FD feel that doctors did not classify them roughly as being "mentally ill." We noticed that no patient clearly expressed his or her opposition to our communication methods. However, the patients may accept the explanation differently due to the different demographic and baseline characteristics (such as gender, education level, GI symptoms, and psychological status). Although not every patient understood and accepted the explanation thoroughly, the feeling of care and attention from doctors during the communication may also relieve their psychological burden and improve treatment satisfaction.

This study also showed that proper communication may improve treatment adherence. Treatment adherence correlated with the decrease of IS and PSS scores ( $r$ values were relatively low because a small portion of patients with very mild pre-treatment stigma showed limited decrease in stigma scores and high compliance). Furthermore, communication may improve treatment efficacy of antidepressants in refractory FD patients. Nearly $90 \%$ of FD patients showed improvement in LDQ scores after antidepres- sant treatment in Group 1. Those with decreased post-treatment stigma scores had even better treatment efficacy. On the contrary, more than one-third of patients with FD in Group 2 had no improvement in symptoms scores. In this study, antidepressants were prescribed to each patient according to their symptoms. Although the mechanisms were not fully illustrated, studies have shown that antidepressants exert actions both in the central nervous system and GI tract. ${ }^{3}$ Antidepressants may have an analgesic effect by interfering with pain-related brain circuits and pain transmission at the level of spinal cord. ${ }^{29,30}$ Furthermore, they could also exert a direct effect on GI function independent of their anxiolytic effects. ${ }^{3}$ The Rome Foundation Working Team suggested that agents acting in both the brain and gut should be relabeled as "gut-brain neuromodulators" to reduce stigma and improve the understanding of neuromodulators. $^{3}$

Besides improving communications regarding the mechanisms of the disease and medications, previous studies have explored other possible strategies to alleviate stigma in patients with mental illness, such as cognitive therapy aimed at altering the stigmatizing beliefs and attitudes of the individual, or enhancing skills for coping with self-stigma. ${ }^{31,32}$ A study in FD patients found that intensified medical management plus cognitive behavior therapy may be effective in alleviating symptoms of depression or anxiety. ${ }^{33}$ However, it is difficult for GI doctors to perform the time-consuming psychological therapy in non-psychological settings. Compared to psychological therapy, proper physician-patient communication may be an easy, efficient, and effective method to prevent and reduce stigma in patients with refractory FD. A number of clinical trials have suggested that reassurance is an important non-pharmacological approach for FD treatment. ${ }^{34-37}$ Reassurance incorporates explanation of the etiology of FD and assurance of the unlikelihood of cancer, which helps to alleviate patients' anxiety and symptoms. ${ }^{34}$ In our study, reassurance was also partly integrated into the communication strategies in Group 1 (explanation of the mechanisms of FD and antidepressants). Assurance of the unlikelihood of cancer and good prognosis of FD should also be added when communicating with FD patients.

There is a limitation in our study. Although we found that FD patients subjected to a proper physician-patient communication had fewer stigmatized attitudes and better treatment compliance after antidepressant treatment, it is still difficult to establish the causality of stigma and treatment compliance.

In conclusion, our results suggest that physicians should pay attention to patients' stigma toward FD and antidepressant medications and reduce stigma by using proper doctor-patient communi- 
cation strategies. This study provides an easy and effective way that could benefit in the treatment of refractory FD via reducing stigma. Improving patients' knowledge of the disease and antidepressant medications through proper physician-patient communication may relieve stigma, improve treatment adherence, and ultimately lead to better treatment efficacy.

\section{Supplementary Material}

Note: To access the supplementary table mentioned in this article, visit the online version of Journal of Neurogastroenterology and Motility at http://www.jnmjournal.org/, and at https://doi. org/10.5056/jnm20239.

Financial support: This work was supported by National Natural Science Foundation of China (Grant No. 81970472 and 81500412 to Xiu-Juan Yan and 81970473, 82170554, 81670484, and 81470812 to Sheng-Liang Chen); and Pujiang Outstanding Youth Project of Renji Hospital South Campus (Grant No. RJPJYQ [2018]).

\section{Conflicts of interest: None.}

Author contributions: Qing-Qing Luo, Bo Wang, Ping Xu, and Chen-Feng Ji enrolled participants and performed the communication; Xiu-Juan Yan and Hong-Yi Qiu analyzed data and prepared manuscript; and Sheng-Liang Chen designed study and reviewed the manuscript.

\section{References}

1. Stanghellini V, Chan FK, Hasler WL, et al. Gastroduodenal Disorders. Gastroenterology 2016;150:1380-1392.

2. Saad RJ, Chey WD. Review article: current and emerging therapies for functional dyspepsia. Aliment Pharmacol Ther 2006;24:475-492.

3. Drossman DA, Tack J, Ford AC, Szigethy E, Törnblom H, Van Oudenhove L. Neuromodulators for functional gastrointestinal disorders (disorders of gut-brain interaction): a rome foundation working team report. Gastroenterology 2018;154:1140-1171, e1.

4. Moayyedi P, Lacy BE, Andrews CN, Enns RA, Howden CW, Vakil N. ACG and CAG clinical guideline: management of dyspepsia. Am J Gastroenterol 2017;112:988-1013.

5. Ford AC, Luthra P, Tack J, Boeckxstaens GE, Moayyedi P, Talley NJ. Efficacy of psychotropic drugs in functional dyspepsia: systematic review and meta-analysis. Gut 2017;66:411-420.

6. Talley NJ, Locke GR, Saito YA, et al. Effect of amitriptyline and escitalopram on functional dyspepsia: a multicenter, randomized controlled study. Gastroenterology 2015;149:340-349, e2.
7. Tack J, Ly HG, Carbone F, et al. Efficacy of mirtazapine in patients with functional dyspepsia and weight loss. Clin Gastroenterol Hepatol 2016;14:385-392, e4.

8. Johnson AC, Greenwood-Van Meerveld B. The pharmacology of visceral pain. Adv Pharmacol 2016;75:273-301.

9. Luo L, Du L, Shen J, Cen M, Dai N. Benefit of small dose antidepressants for functional dyspepsia: experience from a tertiary center in eastern China. Medicine (Baltimore) 2019;98:e17501.

10. Yan XJ, Luo QQ, Qiu HY, Ji CF, Chen SL. The impact of stigma on medication adherence in patients with functional dyspepsia. Neurogastroenterol Motil 2021;33:e13956.

11. Taft TH, Riehl ME, Dowjotas KL, Keefer L. Moving beyond perceptions: internalized stigma in the irritable bowel syndrome. Neurogastroenterol Motil 2014;26:1026-1035.

12. Sirey JA, Bruce ML, Alexopoulos GS, Perlick DA, Friedman SJ, Meyers BS. Stigma as a barrier to recovery: perceived stigma and patientrated severity of illness as predictors of antidepressant drug adherence. Psychiatr Serv 2001;52:1615-1620.

13. Sirey JA, Bruce ML, Alexopoulos GS, et al. Perceived stigma as a predictor of treatment discontinuation in young and older outpatients with depression. Am J Psychiatry 2001;158:479-481.

14. Collins J, Farrall E, Turnbull DA, Hetzel DJ, Holtmann G, Andrews JM. Do we know what patients want? The doctor-patient communication gap in functional gastrointestinal disorders. Clin Gastroenterol Hepatol 2009;7:1252-1254, 1254, e1-e2.

15. Cassell B, Gyawali CP, Kushnir VM, Gott BM, Nix BD, Sayuk GS. Beliefs about GI medications and adherence to pharmacotherapy in functional GI disorder outpatients. Am J Gastroenterol 2015;110:1382-1387.

16. Yeung A, Trinh NH, Chang TE, Fava M. The engagement interview protocol (EIP): improving the acceptance of mental health treatment among Chinese immigrants. Int J Cult Ment Health 2011;4:91-105.

17. Yan XJ, Li WT, Chen X, et al. Effect of clinician-patient communication on compliance with flupentixol-melitracen in functional dyspepsia patients. World J Gastroenterol 2015;21:4652-4659.

18. Singh M. Communication as a bridge to build a sound doctor-patient/ parent relationship. Indian J Pediatr 2016;83:33-37.

19. Shen MJ, Hamann HA, Thomas AJ, Ostroff JS. Association between patient-provider communication and lung cancer stigma. Support Care Cancer 2016;24:2093-2099.

20. Thompson L, McCabe R. The effect of clinician-patient alliance and communication on treatment adherence in mental health care: a systematic review. BMC Psychiatry 2012;12:87.

21. Givens JL, Katz IR, Bellamy S, Holmes WC. Stigma and the acceptability of depression treatments among African Americans and whites. J Gen Intern Med 2007;22:1292-1297.

22. Lysaker PH, Roe D, Yanos PT. Toward understanding the insight paradox: internalized stigma moderates the association between insight and social functioning, hope, and self-esteem among people with schizophrenia spectrum disorders. Schizophr Bull 2007;33:192-199.

23. Moayyedi P, Duffett S, Braunholtz D, et al. The leeds dyspepsia questionnaire: a valid tool for measuring the presence and severity of dyspepsia. Aliment Pharmacol Ther 1998;12:1257-1262. 
24. Herr NR, Williams JW Jr, Benjamin S, McDuffie J. Does this patient have generalized anxiety or panic disorder?: the eational clinical examination systematic review. JAMA 2014;312:78-84.

25. Wang W, Bian Q, Zhao Y, et al. Reliability and validity of the Chinese version of the patient health questionnaire (PHQ-9) in the general population. Gen Hosp Psychiatry 2014;36:539-544.

26. Boskovic J, Leppée M, Culig J, et al. Comparison of two different methods (patient questionnaire and medication possession ratio - MPR) for measuring the chronic patient's behavior. Psychiatr Danub 2014;26(suppl 3):498-508.

27. Looper KJ, Kirmayer LJ. Perceived stigma in functional somatic syndromes and comparable medical conditions. J Psychosom Res 2004;57:373-378

28. Qin HY, Cheng CW, Tang XD, Bian ZX. Impact of psychological stress on irritable bowel syndrome. World J Gastroenterol 2014;20:1412614131.

29. Atkinson JH, Slater MA, Wahlgren DR, et al. Effects of noradrenergic and serotonergic antidepressants on chronic low back pain intensity. Pain 1999;83:137-145.

30. Jackson JL, Shimeall W, Sessums L, et al. Tricyclic antidepressants and headaches: systematic review and meta-analysis. BMJ 2010;341:c5222.

31. Mittal D, Sullivan G, Chekuri L, Allee E, Corrigan PW. Empirical studies of self-stigma reduction strategies: a critical review of the literature. Psychiatr Serv 2012;63:974-981.

32. Hansson L, Lexén A, Holmén J. The effectiveness of narrative enhancement and cognitive therapy: a randomized controlled study of a selfstigma intervention. Soc Psychiatry Psychiatr Epidemiol 2017;52:14151423.

33. Haag S, Senf W, Tagay S, et al. Is there a benefit from intensified medi$\mathrm{cal}$ and psychological interventions in patients with functional dyspepsia not responding to conventional therapy? Aliment Pharmacol Ther 2007;25:973-986.

34. Wang YP, Herndon CC, Lu CL. Non-pharmacological approach in the management of functional dyspepsia. J Neurogastroenterol Motil 2020;26:6-15

35. Vanheel H, Tack J. Therapeutic options for functional dyspepsia. Dig Dis 2014;32:230-234.

36. Enck P, Klosterhalfen S. The placebo response in functional bowel disorders: perspectives and putative mechanisms. Neurogastroenterol Motil 2005; 17:325-331.

37. Moayyedi P, Soo S, Deeks J, Delaney B, Innes M, Forman D. Pharmacological interventions for non-ulcer dyspepsia. Cochrane Database Syst Rev 2006:CD001960. 